\title{
Neural Networks Optimization, Using the Genetic Algorithm
}

\author{
Somaieh Khajeh Hasani Rabori ${ }^{1}$, Khojaste Shour bakhloo \\ ${ }^{1,2}$ Sirjan Technical University
}

\begin{abstract}
There are many ways in neural networks optimization by genetic algorithm. One of the optimization methods is the neural networks repetition. The main idea of this method is that the model is developed at first and then trained using the less trained samples and probable searching area respectively. Approved results based on applied cases has been added as the new model training and second method is the genetic algorithm addition to the neural network structure and weights. In this optimization the weight, Bios and the neural networks structure are considered for awareness. The presented method application is assessed in time series prediction flaws. Other method is the optimization of scale neural network by the genetic algorithm. This method is used a granular method based on database complexity. Presented method has been tested by the face identification problem. People distribution in each granule with an initial analysis has been determined, caused the classification of data via the similar complexity.
\end{abstract}

Keywords: optimization, neural networks, genetic algorithm, forming, time series prediction

\section{Introduction}

Most of engineering problems such as system designing, process control, forecasting, making the parts are the sections of optimization. The aim of engineering optimization generally is the system operation improvement, increasing of process control and accuracy forecasting, production quality improvement and saving the costs. Considering that written functions of system input and output are mainly complex and nonlinear, therefore it is impossible to write an exact solution. So some of modelling methods based on approximation such as response surface, base radial function, Kriging model and Neural Networks are used generally for explicit functions approximation in waste domain of engineering applications. Among these methods the Neural Network is an index for the nonlinear multivariable system modelling. During the recent years the Artificial Neural Network training has been increased and the nonlinearity is the main reason for it. Choosing the layer, node and nodes in each layer is the most challengeable subject. In this situation an affordable ANN model is needed. Sekton et al were used the GA and Simulated Annealing for this aim. Among the ANN methods the BPNN is the most classic training algorithm that used considerably. Actually it is a feed forward multi-layer that trained by the BP algorithm. This does not guarantee the optimized answer and also has the little rate of convergence. However the ANN needs to be assessed for the noise certainty and the researchers found that BPNN and GA combination is an acceptable method for this method optimization.

\section{Optimization Approach}

Genetic algorithm is a random and exploration optimization based on genetic principles and revolution theory. Actually it is a forward research approach that moves rapidly towards the best solution finding. The neural network can be optimized by the GA in many ways. Figure one shows the suggested optimization of GA and BPNN integration repetition. 


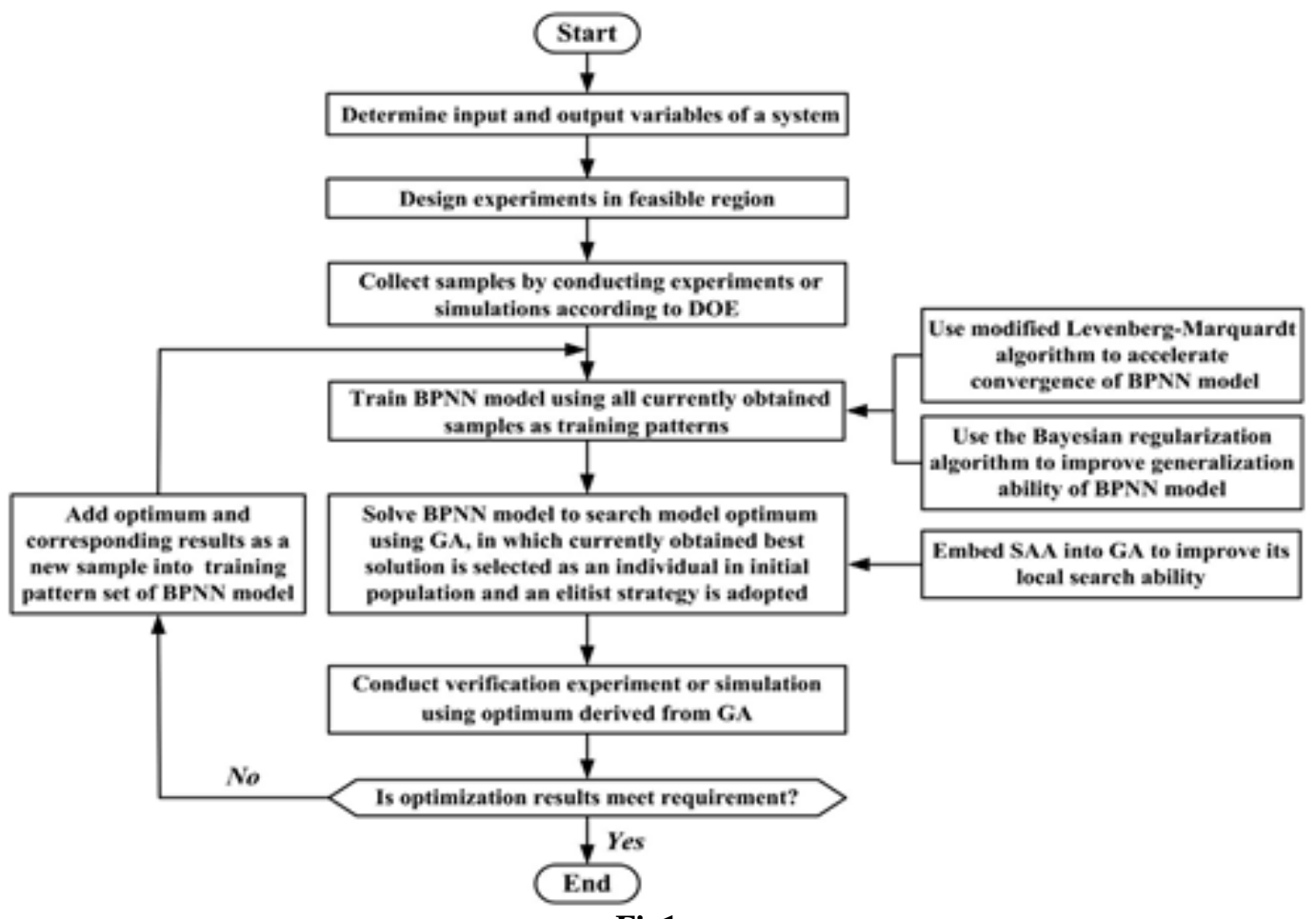

The main idea of optimization is as below:

At first BPNN is run and is trained by the samples with fewer training. Then by the trained BPNN model and suitable GA the model will be solved for the probable area and will be optimized. After that the empirical results and desired simulations will be added to the BPNN as the new training sample. Therefore one simulation is needed in each repetition. The training and research process will be continued until the optimization process converged. For the prevention of accuracy reduction 4 strategy has been legislated.

1. The Bayesian setting algorithm is used during the BPNN model training for the decision making improvement.

2. Modified Levenberg-Marquardt training algorithm is used for the BPNN convergence acceleration.

3. Simulated annealing algorithm usage in GA, improves the local research ability.

4. For each repetition the best solution always chooses a case in initial population.

Table1. Empirical designing by L18 Orthogonal Array and objective function amounts Y

\begin{tabular}{|l|l|l|l|l|l|l|l|l|}
\hline Exp. No. & O1 & O2 & O3 & O4 & O5 & O6 & O7 & y \\
\hline 1 & 65 & 65 & 65 & 65 & 65 & 65 & 65 & 0.0144 \\
\hline 2 & 65 & 80 & 80 & 80 & 80 & 80 & 80 & 0.0120 \\
\hline 3 & 65 & 95 & 95 & 95 & 95 & 95 & 95 & 0.1455 \\
\hline 4 & 80 & 65 & 65 & 80 & 80 & 95 & 65 & 0.0355 \\
\hline 5 & 80 & 80 & 80 & 95 & 95 & 65 & 65 & 0.0988 \\
\hline 6 & 80 & 95 & 95 & 65 & 65 & 80 & 80 & 0.0135 \\
\hline 7 & 95 & 65 & 80 & 65 & 95 & 80 & 95 & 0.0322 \\
\hline 8 & 95 & 80 & 95 & 80 & 65 & 95 & 65 & 0.0307 \\
\hline 9 & 95 & 95 & 65 & 95 & 80 & 65 & 80 & 0.1631 \\
\hline 10 & 65 & 65 & 95 & 95 & 80 & 80 & 65 & 0.2451 \\
\hline 11 & 65 & 80 & 65 & 65 & 95 & 95 & 80 & 0.0787 \\
\hline 12 & 65 & 95 & 80 & 80 & 65 & 65 & 95 & 0.0397 \\
\hline 13 & 80 & 65 & 80 & 95 & 65 & 95 & 80 & 0.0082 \\
\hline 14 & 80 & 80 & 95 & 65 & 80 & 65 & 95 & 0.0556 \\
\hline 15 & 80 & 95 & 65 & 80 & 95 & 80 & 65 & 0.0851 \\
\hline 16 & 95 & 65 & 95 & 80 & 95 & 65 & 80 & 0.0992 \\
\hline 17 & 95 & 80 & 65 & 95 & 65 & 80 & 95 & 0.0152 \\
\hline 18 & 95 & 95 & 80 & 65 & 80 & 95 & 65 & 0.0191 \\
\hline
\end{tabular}

Suggested method is applied on products wall thickness for the Neural Network optimization. Optimized parameter setting affects the efficiency, quality, production cost and delivery time. Molding and Making Extrusion(EBM) are one of the main technology in polymer production. In this method the melted 
polymer via a Parison mold is located as a circular Parison mold. The aim of this method optimizing is the usage of suggested method and finding the optimum mold gap in 9 point of produced teeth. Figure 2 shows the optimization flowchart in details.

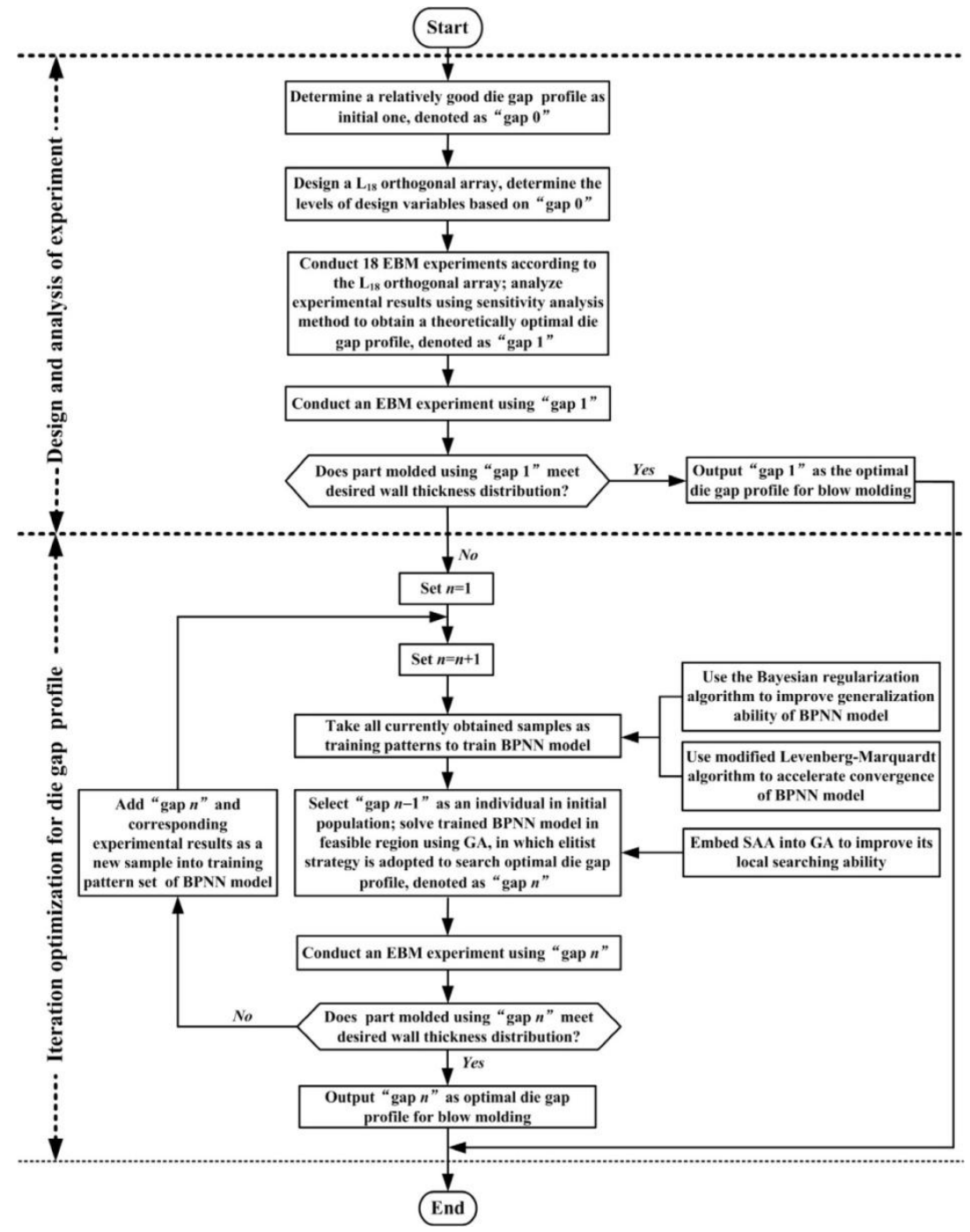

Fig2. Optimization flowchart

Optimization consist of two part, designing and analyzing of the experiment and repetition optimization for the gap properties. In first step, designing and analyzing, the orthogonal designing has been used in more effective indices gathering in BPNN training model. The designing variable surfaces of initial mold gap is determined with an opening constant equal to 80\%. In 3 surface for each variable the designing was done After the 18 trial (table1).

The results of orthogonal experiment are analyzed, using the sensitivity analysis. After that the optimum level of each designed variable will be analyzed and corresponding to gap properties a distance will determined, for example distance 1. The verification of "distance 1" will be done and the thickness will be indicated in 9 point. If the intended thickness obtained, the output of distance 1 is chosen as the optimum mold gap for the molding process. In optimization the repetition for the gap properties of all samples is used as the BPNN training pattern. Three layer (input, hidden and output layer) has been developed for the BPNN model. The Sigmoid and Linear transformation functions are used for the hidden and output layers respectively. Seven mold aperture in all samples have been selected as the input parameters for BPNN model. Since there is no any exact rule in neuron numbers indication, therefore the trial and error method is used for this aim. The results have shown that BPNN model works optimum with 10 neuron in hidden layer. The BPNN training has been done, using the Bayesian and modified Levenberg-Marquardt. The trained BPNN model can be used for the assessment of the objective function in set of designed variables in probable area. Therefore the trained BPNN model is used directly as the GA fitness function for the model solution search. For each gap optimization the "1-N gap" is used as the initial population of GA. Actually the GA as a research method is implemented in 4 
step: Initialized, Selection, Permutation and Mutation. The neural networks model can be optimized, using a novel solution for genetic algorithm. The suggested model operation is evaluated in time series prediction problems. The results show the excellence of this method usage comparing to the other methods. The researcher used the Taboo search and simulated annealing for the optimization of weight and nodes in neural networks. After that they followed their studies, utilizing the hybridization of taboo search, simulated annealing and the GA that resulted in GRNN model introducing, a genetic algorithm based on dynamic neural network. This method provides the checking opportunity of various weights, Bios, hidden layer numbers, the number of nodes and selected inputs during the search process. In this method each solution is presented via two one dimension vector. The first vector belongs to the solution structure and second vector belongs to the weight and neural network bios. Each cell in solution structure has the 0 or 1 value while it has a wide range between 1 and -1 for the weight and solutions. In structure solution three cell are considered for hidden layer and three more cell for the nodes number in each of hidden layers. The network structure sketch has been done in two step: 1- the no optimized training was done and new determination numbers were determined for each recognition. 2- The hierarchy genetic algorithm has been used.

\section{Conclusion}

In present study it has been tried to express the various methods of optimization. The integrated hybrid optimization method, using the BPNN and GA has been presented. Suggested method prevents the Caught in local optimums. On the other hand the BPNN training can be done by several training samples. The trained BPNN after that was used as fitness function of GA for the search model solving. Results show that suggested method is applicable and effective in finding the optimum gap properties, reaching the intendedthickness distribution. Suggested method can help effectively in production quality and costs. Neural network optimization via the simultaneous weight and bios of GRNN model will approve the perfect results in rate and network error. Indeed the optimization can provide the working with huge databases that can be used for training phase.

\section{References}

[1] NS Jaddi, S Abdullah, AR Hamdan - Information Processing Letters, 2016 - A solution representation of genetic algorithm for neural network weights and structure. ElsevierCamisón,

[2] S.I. Chang, K.P. Seng, L. Ang, 2012, Modular dynamic RBF neural network for face recognition, in: IEEE

[3] Conference on Open Systems (ICOS), pp. 1-6.

[4] HX Huang, JC Li, CL Xiao - Expert Systems with Applications, 2015 - A proposed iteration optimization approach integrating backpropagation neural network with genetic algorithm.ElsevierHazen, 